\title{
Combination Law for Drude- Sommerfeld's Electron Damping in Multilayer Thin Metal Films
}

\author{
Stefano A. Mezzasalma*, Vesna Janicki, Krešimir Salamon, and Jordi Sancho-Parramon*
}

Materials Physics Division, Ruđer Bošković Institute, Bijenička cesta 54, 10000 Zagreb, HR

Key words: optical thin metal films, electron damping, $\mathrm{Cu} / \mathrm{Au}$ and $\mathrm{Au} / \mathrm{Ag}$ multilayers, Drude-Sommerfeld model

*Corresponding author: e-mail Stefano.Mezzasalma@irb.hr, Jordi.Sancho.Parramon@irb.hr

A combination rule for electron $\left(\mathrm{e}^{-}\right)$damping in multilayer thin metal films is derived from a mean-field picture and is applied to optical experimental data. The overall coefficient obeys a parallel law of pure materials damping, $\langle\gamma\rangle^{-1}=\bar{g}_{A} \gamma_{A}^{-1}+\bar{g}_{B} \gamma_{B}^{-1}\left(\bar{g}_{i}<1\right)$, chemical specificity being involved by averaging over densities of low energy states in the free $\mathrm{e}^{-}$model. Geometric and static electromagnetic features of single layers couple via small Fermi's energy fractions $\left(\alpha_{i}\right)$. An application is developed for thin $\mathrm{Cu} / \mathrm{Au}$ and $\mathrm{Au} / \mathrm{Ag}$ films, showing an apparently irregular damping trend in the film thickness $\left(d_{i}=2.5-7.5 \mathbf{n m}\right)$. The inferred $\langle\gamma\rangle$ 's agree in both cases with our data when $\left|\alpha_{i}\right| \approx 10^{-2} \div 10^{-3}$ linearly increases with decreasing $d_{i}$, suggesting a coupling phenomenology that can bring new insights in the optics and plasmonics of nanostructures.

Copyright line will be provided by the publisher

Bimetallic systems are the subject of relevant nanoscience investigations, among which the optical response of nanostructures of group 11 noble metals, e.g. $\mathrm{Au}, \mathrm{Ag}, \mathrm{Cu}$ [1-3]. Their polarizability, promoted by $d^{10}$ electrons $\left(\mathrm{e}^{-}\right)$and large free $\mathrm{e}^{-}$densities, entitle them to be the preferred candidates for plasmonics applications in the visible light spectrum. Alloyed nanomaterials can show functionalities (e.g. catalysis, energy storage and optics) lying beyond those of their constituents, as the $\mathrm{e}^{-}$binding and charge distributions in the alloy alter the electronic (surface) properties of pure metals and, accordingly, their chemical behavior $[4,5]$. To improve the versatility of plasmonic materials, the surface plasmon resonance characteristics thus are engineered by combining (i.e. alloying, layering, intermixing) metal particles of different kinds [6, 7]. By focusing on layered systems, core-shell nanoparticles were shown to provide superior Raman enhancement [8], improved antibacterial properties [9] and larger tunable chiral response [10] than nanoparticles made by pure materials. Segregated bimetallics or Janus nanoparticles then offer a highly tailorable optical response, as it comes from their geometric and chemical asymmetry [11], and improved electrocatalytic features [12]. Apart from their use as optical coatings in X-ray spectral ranges [13,14], 2-dimensional bimetallic multilayer systems can jointly furnish mechanical and electrical features that are unattainable with pure materials [15]. Optical features of metal films depend on deposition history, thickness, strains, grain size, texture and crystallinity degree [16]. This uncertainty implies the relative permittivity of mixtures to be measured at specified compositions, and afterwards compared to the available models [17]. Four are the main levels at which metals can be modeled: perfect conductors, dispersive materials, dissipative materials within classical Drude's model [18] and extended Drude's model [19], when the carrier lifetime is frequency-dependent. Drude's theory, thus, still applies in many cases to model the low-frequency absorption domain, affording a straightforward explanation of ion core- $\mathrm{e}^{-}$interactions by an effective $\mathrm{e}^{-}$mass and the sign of carrier charges [20]. Modeling dielectric properties of material mixtures, however, may become a tough task. Effective medium approximations alone may be used [21], provided the sizes of mixing phases are smaller than the light wavelength, but are large enough to preserve the dielectric information of reference media. In alloys, they disregard possible structural changes and therefore fail even though permittivity values of pure metals are accurately known beforehand [1]. Dielectric functions are unpredictable too from composition-weighted averages of pure constituents, as it was shown for $\mathrm{Au} / \mathrm{Ag}[22,23]$. In 
well-separated layered media, effective medium theories may be affected by non-locality and size confinement [24]. This work focuses on the effective $\mathrm{e}^{-}$damping in thin multilayer metal films. Damping is the most critical parameter ultimately determining the quality factor in many optics and plasmonics applications [25,26]. By combining a mean-field view with the free $\mathrm{e}^{-}$model, the overall behavior fulfills a parallel combination rule of pure materials coefficients, as here it is tested for $\mathrm{Cu} / \mathrm{Au}$ and $\mathrm{Au} / \mathrm{Ag}$. Theories linking mesoscopic to microscopic/atomic scales well suit condensed matter investigations such as polarization, dielectric [27-29], flow [30] and solid-state properties [31]. This picture can allow a quantitative analysis of nonlocal processes in bimetal samples, fruitful to study and design plasmonic devices relying on thin layered media.

Experimental Two types of multilayer structures were produced and studied, $\mathrm{Cu} / \mathrm{Au}$ and $\mathrm{Au} / \mathrm{Ag}$. Optical features of all pure materials are nicely described by Drude's model in spectral regions for photon energies $<1.5 \mathrm{eV}$ [32], where interband transitions are absent and $\mathrm{e}^{-}$damping coefficients follow from optical spectroscopy methods in the near-infrared range [33]. Due to good wetting properties, $\mathrm{Cu}$ is less prone than $\mathrm{Ag}$ and $\mathrm{Au}$ to form islands when it is deposited onto a dielectric or semiconductor surface [34]. To improve Au wetting, substrates were precoated by a $5 \mathrm{~nm}$ thick Cr layer [35]. By using electron beam evaporation, a number of $\mathrm{Cu} / \mathrm{Au}$ and $\mathrm{Au} / \mathrm{Ag}$ multilayers were deposited on a silicon wafer, $\operatorname{Si}(100)$, comprising 5 pairs of $\mathrm{Cu} / \mathrm{Au}$ and $\mathrm{Au} / \mathrm{Ag}$ layers with different combinations of metal thicknesses. Depositions were made in a modified Varian chamber with base pressure of $6 \times 10^{-7}$ Torr, where a quartz crystal controller monitored the thickness of metal layers, growing with rate $\approx 1 \AA / \mathrm{s}$. Substrates weren't preheated before deposition to prevent from metal island formations and get compact films.

$\mathrm{X}$-ray reflectivity (XRR) spectra (in Supporting Information A) were detected to control the fabricated multilayer structures. They were carried out by a diffractometer equipped with a Co X-ray tube and a position-sensitive detector (Hecus PSD-50M) that was placed $500 \mathrm{~mm}$ from the samples. XRR scans were obtained by measuring the intensity of specularly reflected X-ray beams as a function of the grazing incidence angle (ranging from $0^{\circ}$ to $1.2^{\circ}$ with step $0.003^{\circ}$ ). In all examined multilayers, XRR data showed Bragg peaks and oscillations that confirmed the structure periodicity and thickness uniformity. Best fits were calculated by the standard Parratt algorithm taking roughness into account according to Nevot and Croce [36], revealing thickness values in close agreeement with those detected by the quartz control and densities of layers agreeing as well with their reference values.

Optical measurements allowed retrieving the effective dielectric function and thickness of the whole multilayer. Ellipsometric data were obtained in the spectral range $(275-2175) \mathrm{nm}$ by a J.A. Woollam V-VASE ellipsometer at incidence angles of $65^{\circ}, 70^{\circ}, 75^{\circ}$. Dielectric functions
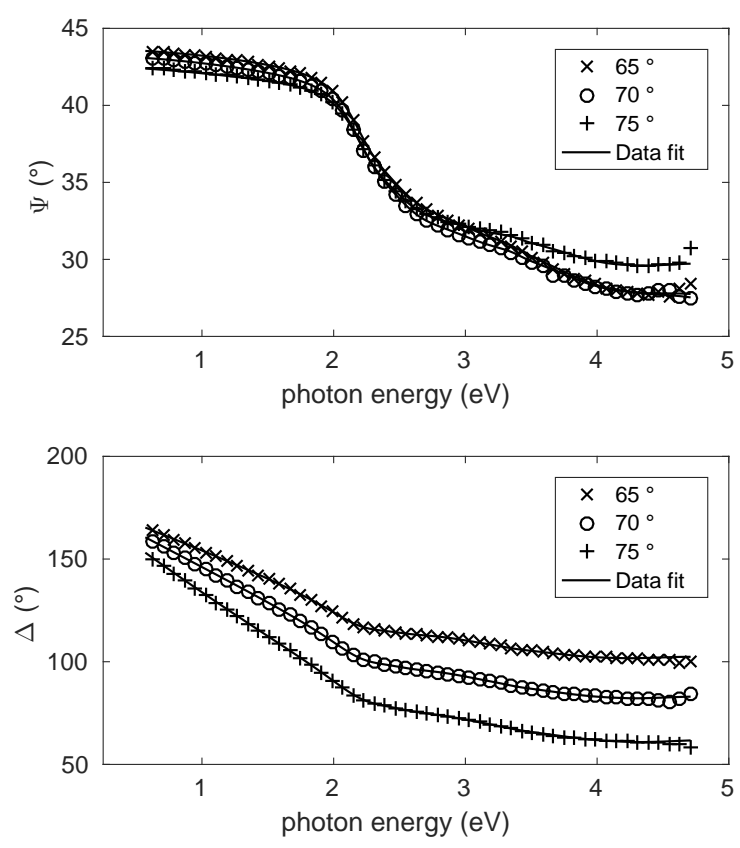

Figure 1 Ellipsometric angles (symbols) and best fits (solid lines) from a multiple-oscillator model for effective optical constants of $\mathrm{Cu} / \mathrm{Au}(5.0 / 5.0)^{5}$. Data for $\mathrm{Au} / \mathrm{Ag}$ are illustrated in Supporting Information B. Quality of this fit is representative of the two entire data sets. Legends also report the incidence angle.

of composites followed from a multiple-oscillator model, taking both interband transitions in the visible-UV spectral range and low-energy Drude's response into account [34]. Data were best fitted by optimizing the dispersion parameters minimizing the discrepancy among experimental and simulated spectra. This method is implemented by the WVASE software and always led to excellent fits in the entire spectral range. Figures 1 and 2 illustrate typical responses for $\mathrm{Cu} / \mathrm{Au}$ systems, $\mathrm{Au} / \mathrm{Ag}$ 's being depicted in Supporting Information B. The good fit quality in all cases implies that multilayers can be well described by homogeneous media with effective optical constants, obeying Drude's model in the low-energy region (Fig. 2). Effective thicknesses were set to the total multilayer's.

A synopsis of relevant data is drawn in Table (1). Drude's plasma frequencies $\left(\omega_{P}\right)$ did not show relevant changes in all samples, with mean values $\langle\omega\rangle_{P}=8.62$, $8.80 \mathrm{eV}$ and relative $\%$ deviations $\approx 3.5,2$, respectively in $\mathrm{Cu} / \mathrm{Au}$ and $\mathrm{Au} / \mathrm{Ag}$. Optical parameters of pure metals agree with former plasma frequency values and damping coefficients of $\mathrm{Au}, \mathrm{Ag}$ and $\mathrm{Cu}$ [37-40]. Owing to slightly different deposition conditions, Au data turned out to differ in the two multilayer series. Note that samples may exhibit an electromagnetically anisotropic behavior. Our measurements, however, reflect the response to an electric field directed parallelly to each multilayer structure. The 


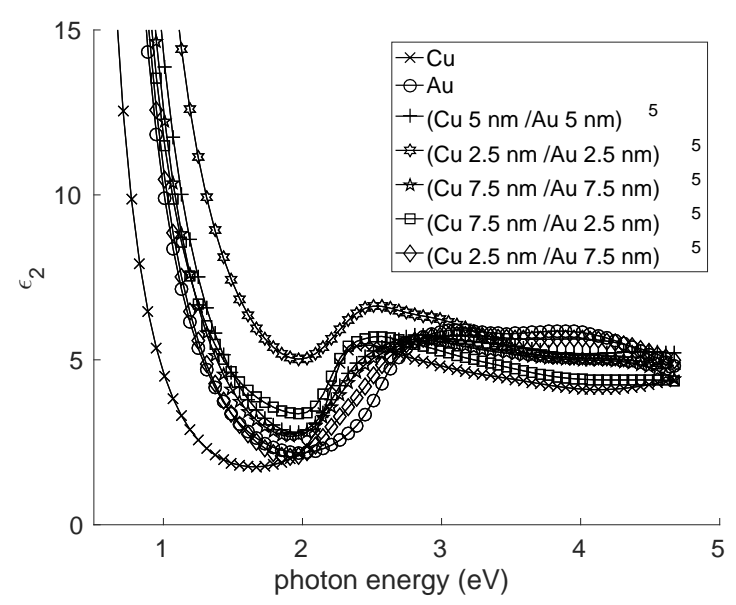

Figure 2 Imaginary part of the effective dielectric function $(\epsilon)$ of $\mathrm{Cu} / \mathrm{Au}$ samples (Au/Ag's is shown in Supporting Information B).

Table 1 Geometrical and Damping Data $(\mathrm{k}=$ bilayer number).

\begin{tabular}{lll}
\hline${\text { thicknesses }(\mathrm{nm} / \mathrm{nm})^{k}}^{k} \mathrm{Cu} / \mathrm{Au}(\mathrm{meV})$ & $\mathrm{Au} / \mathrm{Ag}(\mathrm{meV})$ \\
\hline$(35 / 0)^{1}$ & 136 & 96 \\
$(0 / 35)^{1}$ & 69 & 62 \\
$(5.0 / 5.0)^{5}$ & 196 & 120 \\
$(2.5 / 2.5)^{5}$ & 281 & 142 \\
$(7.5 / 7.5)^{5}$ & 168 & 98 \\
$(2.5 / 7.5)^{5}$ & 163 & 123 \\
$(7.5 / 2.5)^{5}$ & 138 & 110 \\
\hline
\end{tabular}

continuity condition for displacement vectors at the airmetal interface strongly attenuates the longitudinal field component in the layer, as in metals the dielectric function is large in most of the spectral range [41].

Theoretical Drude's relation for charge displacement in a metal $i$ is a function of mass $\left(m_{i}\right)$, electric charge $\left(q_{i}\right)$, damping coefficient $\left(\gamma_{i}\right)$, electric field intensity $\left(\mathrm{E}_{i}\right)$ and frequency $(\omega)$ as:

$$
r_{i}=-\frac{q_{i} \mathrm{E}_{i}}{m_{i} \omega_{i}\left(\omega_{i}+\mathrm{i} \gamma_{i}\right)}
$$

Equation (1) follows from a Lagrangian view for a dissipative $n$-body system, with vector positions $\mathbf{r}_{i}(i \leq n)$ in a region with external electric field $(\phi)$, classical potential (U) and kinetic $(\mathrm{K})$ energies, as well as Rayleigh's dissipation function $\left(\mathrm{D}_{R}\right)$. For $n=2(i=A, B)$ [42]:

$$
\begin{gathered}
\mathrm{U}=q_{A} \phi\left(\mathbf{r}_{A}\right)+q_{B} \phi\left(\mathbf{r}_{B}\right)+k_{c} \frac{q_{A} q_{B}}{|\mathbf{r}|} \\
\mathrm{K}=\frac{1}{2} m|\mathbf{v}|^{2}+\frac{1}{2} M|\overline{\mathbf{v}}|^{2} \\
\mathrm{D}_{R}=\frac{m_{A}}{2} \gamma_{A}\left|\mathbf{v}_{A}\right|^{2}+\frac{m_{B}}{2} \gamma_{B}\left|\mathbf{v}_{B}\right|^{2}
\end{gathered}
$$

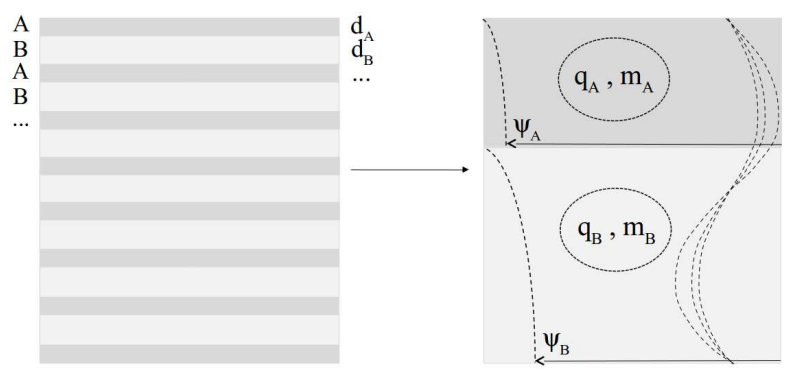

Figure 3 Scheme of the mean-field approach, $\psi_{i}, m_{i}, q_{i}$, denoting the $i$-th effective field attenuation (Eq. 9), mass and charge.

with total and reduced masses, $M$ and $m=m_{A} m_{B} / M$, velocities of baricentre $(\overline{\mathbf{r}})$ and the position difference $(\mathbf{r})$, $\overline{\mathbf{v}}=p_{A} \mathbf{v}_{A}+p_{B} \mathbf{v}_{B}\left(p_{i}=m_{i} / M\right)$ and $\mathbf{v}=\mathbf{v}_{B}-\mathbf{v}_{A}\left(k_{c}\right.$ is Coulomb's constant). Overwritten symbols will mean onward mass averages, in the fashion of $\overline{\mathbf{v}}$. Equation (1) stems from Euler-Lagrange's once electric fields $\mathbf{E}_{i}=\mathrm{E}_{i} \mathrm{e}^{-\mathrm{i} \omega t} \mathbf{e}_{\mathrm{t}}$ and charge displacements $\mathbf{r}_{i}=r_{i} \mathrm{e}^{-\mathrm{i} \omega t} \mathbf{e}_{\mathrm{t}}$ are adopted, $\mathrm{E}_{i}$ being space-dependent and $\mathbf{e}_{t}$ denoting the unit vector in transverse direction. For longitudinal Coulomb's forces, one gets in fact an equation system just solved by Eq. (1):

$$
\left(\begin{array}{cc}
\Omega_{A} & \Omega_{B} \\
\widehat{\Omega}_{A} & \widehat{\Omega}_{B}
\end{array}\right)\left(\begin{array}{c}
r_{A} \\
r_{B}
\end{array}\right)=-\left(\begin{array}{c}
\frac{q_{A}}{M} \mathrm{E}_{A}+\frac{q_{B}}{M} \mathrm{E}_{B} \\
\frac{q_{B}}{m_{B}} \mathrm{E}_{B}-\frac{q_{A}}{m_{A}} \mathrm{E}_{A}
\end{array}\right)
$$

with $\Omega_{k} / p_{k}=\mathrm{i} \omega\left(\gamma_{k}-\mathrm{i} \omega\right)=\mp \widehat{\Omega}_{k}(k=A, B)$. Supporting Information $\mathrm{C}$ reports the main mathematical details.

On this basis, a mean-field approach is stated in the following for $\mathrm{e}^{-}$damping in a bilayer of distinct metals. Geometry is regarded infinite in the horizontal plane with thicknesses at the nanoscale, each layer carrying an overall mass and charge (Fig. 3). As Drude's model well reproduced all data, no other dissipation source was added to $\mathrm{D}_{R}$ to include e.g. mean free path constraints from interfaces or grain boundaries [43]. Drude's dielectric functions come from inserting the $\left(\mathrm{e}^{-}\right)$charge displacement $\left(\mathbf{r}_{i}\right)$ in the displacement field by the polarization vector density. An akin analysis can be made for overall displacements obeying a weighted average like:

$$
\langle r\rangle=\varrho_{A} r_{A}+\varrho_{B} r_{B}
$$

its weights $\left(\varrho_{i}\right)$ being defined through $\langle r\rangle=\left\langle r^{*}\right\rangle$, where:

$$
\left\langle r^{*}\right\rangle=\frac{-q \mathrm{E}_{\mathbf{r}}}{M \omega(\omega+\mathrm{i}\langle\gamma\rangle)}
$$

Now, overall charge, electric field and damping coefficient, $q, \mathrm{E}_{\mathbf{r}}$ and $\langle\gamma\rangle$, can be linked to the same quantities in single layers by suitable partitioning coefficients. Let $n_{i}$ be the $i$ th $\mathrm{e}^{-}$density, charge fractions obey $q_{i} / q=p_{i} \pi_{i} / \bar{\pi} \equiv \chi_{i}$, with $\pi_{i}=n_{i} / \rho_{i}$ being the charge amount per unit mass ( $\rho=$ mass density per unit volume). Field attenuations are 
equally needed in each layer, thus we define $\mathrm{E}_{i} / \mathrm{E}_{\mathbf{r}} \equiv \psi_{i}$. Replacing such partition terms in Eq. (6) and Eq. (7) yields:

$$
\varphi_{A} \widehat{\psi}_{A}+\varphi_{B} \widehat{\psi}_{B}=\varphi
$$

with $\varphi_{i}=p_{i} \pi_{i}^{2}, \varphi=\bar{\pi}^{2}$ and:

$$
\widehat{\psi}_{i}\left(\psi_{i}, \omega\right)=\frac{\omega+\mathrm{i}\langle\gamma\rangle}{\omega+\mathrm{i} \gamma_{i}} \psi_{i}
$$

where $\psi_{i}=\psi\left(d_{i}, \omega\right)$ is function of the thickness $d_{i}$. Short notes on the pure materials behavior in Eq. (8) are reported in Supporting Information D. To express $\psi_{i}$, we remind no static longitudinal fields establish in conducting media without applied current densities. In good conductors, the lowest-order propagation vector in $\omega \epsilon_{i} / \sigma_{i} \ll 1$ is $k_{i \perp} \approx(1+\mathrm{i}) \sqrt{\widetilde{\mu}_{i} \sigma_{i} \omega / 2}$, where $\epsilon$ is the dielectric permittivity, $\sigma$ is the electric conductivity, $\widetilde{\mu}$ is the magnetic permeability [41]. As thicknesses relate to metal volume fractions $\left(d_{i} / d_{j} \rightarrow 0\right.$ iff $\left.p_{i} \rightarrow 0\right)$, pure materials responses should be recovered as $p_{i} \rightarrow 1$ or $d_{i} \rightarrow \infty$. A way to impose this is through the field decay across each layer, i.e. $\psi_{i}\left(d_{i}, \omega\right) \approx 1-\mathrm{e}^{-\operatorname{Im}\left\{\mathrm{k}_{\mathrm{i}} \perp(\omega)\right\} d_{i}}$, giving an intrinsic decay contribution from layer $i$. If $d_{i} \rightarrow 0$ then $\psi_{i} \rightarrow 0$ and, correspondingly, $p_{i} \rightarrow 0$ (and vice versa if $d_{i} \rightarrow \infty$ ).

To involve the individual metal features, Eq. (8) may be averaged over the densities of occupied states $D_{i}=D_{i}(\varepsilon)$ $[44,45]$, reminding that Drude's intraband transitions are low-energy's. For any frequency function in the layer $i$, $I_{i}=I_{i}(\varepsilon / \hbar)(\hbar=$ Planck's constant $)$, the operator:

$$
\left\langle I_{i}\right\rangle=\frac{C_{i}}{n_{i}} \int_{0}^{\infty} D_{i} I_{i}(\varepsilon) d \varepsilon
$$

is calculated to let the phenomenological behavior of each damping contribution emerge from Eq. (8) i.e.:

$$
\varphi_{A}\left\langle\widehat{\psi}_{A}\right\rangle+\varphi_{B}\left\langle\widehat{\psi}_{B}\right\rangle=\varphi
$$

As temperature effects aren't regarded here, the factor $C_{i}$ will normalize any thermal coefficient to recover the single layer responses in all of the involved physical quantities. In particular, Eq. (11) will be coupled to Drude's low-energy domain by resorting to Sommerfeld's expansion [44]:

$$
\int_{0}^{\infty} f_{D i} I_{i}(\varepsilon) d \varepsilon \approx \int_{0}^{\mu_{i}} I_{i}(\varepsilon) d \varepsilon+\frac{\pi^{2}}{6}\left(k_{B} T\right)^{2} I_{i}^{\prime}\left(\mu_{i}\right)
$$

where $k_{B} T$ is Boltzmann's thermal factor, prime is the first $\varepsilon$-derivative, $f_{D i}\left(\varepsilon, \mu_{i}\right)$ is Fermi-Dirac's distribution function with chemical potential $\mu_{i}$, entering $D_{i}=A_{e} \sqrt{\varepsilon} f_{D i}$ with $2 \pi^{2} A_{e}=\left(2 m_{e} / \hbar^{2}\right)^{\frac{3}{2}}$ ( $m_{e}=\mathrm{e}^{-}$mass). Equation (11) is averaged upon $\mu_{i} \approx \varepsilon_{i F}$ (Fermi's energy), valid at low $T$, and introducing Fermi's energy fractions through $\delta \varepsilon_{i} / \varepsilon_{i F} \equiv \alpha_{i} \ll 1$. Calculations, reported in Supporting Information $\mathrm{E}$, return an overall damping coefficient of the form:

$$
\bar{g}\langle\gamma\rangle^{-1}=g_{A} \gamma_{A}^{-1}+g_{B} \gamma_{B}^{-1}
$$

with dimensionless coefficients:

$$
g_{i}=\frac{\varphi_{i}}{\varphi} \alpha_{i}^{2} \lambda_{i} \sqrt{\varepsilon_{i F}}, \bar{g} \equiv \overline{\alpha^{2} \lambda \varepsilon_{F}}
$$

and where $\lambda_{i}=d_{i} \sqrt{\widetilde{\mu}_{i} \sigma_{i} / 2}$ is coupling the layer thickness to electromagnetic features of each phase. Note that pure dampings recover identically from Eq. (13) upon $p_{i} \rightarrow 1$.

Results and Discussion Equation (13) shows a parallel combination law for Drude's $\mathrm{e}^{-}$damping in multilayer thin films. Coefficients can obey $g_{i} /\langle g\rangle<1$, a condition allowing larger damping (smaller relaxation times) from a parallel (series) rule. It quantitatively explain two dissimilar data series, the first of which e.g. damping less when $\mathrm{Cu} / \mathrm{Au}=7.5 / 2.5$ and 2.5/7.5, than 7.5/7.5 and 5/5. These trends aren't anyway explained by effective medium theories for metals, as optical parameters would range in this case between pure materials values (see the numerical analysis in Supporting Information F). Qualitatively, a close situation arises from thin alloyed materials (see e.g [46] Au/Ag), in compliance with Matthiessen's rule [47]. Dependence of weight coefficients on $\sqrt{\varepsilon_{F}}$ agree with the prediction of larger damping with increasing Fermi's velocity, $\Delta \gamma \sim v_{F} / L[48,49]$ ( $L=$ length scale). Note that, in original Drude's assumptions, $\gamma$ is independent of $\varepsilon$, while the more realistic assumption of energy-independent mean free path (scattering e.g. from lattice vibrations or neutral impurities) would set $\gamma \propto \sqrt{\varepsilon}$ [50]. The quantities $\alpha_{i} \ll 1$ couple the sum over states to low energies, where Drude's response takes place. They specialize the analysis by defining an energy slice for each metal. This approach acts as an optical extrapolation for good conducting layers, from very low to moderately low energies.

Our data, $\gamma_{A}=136 \mathrm{meV}, \gamma_{B}=69 \mathrm{meV}$ and $\gamma_{A}=96$ $\mathrm{meV}, \gamma_{B^{\prime}}=62 \mathrm{meV}$ were taken as pairwise reference states for Eq. (13) when $A \equiv \mathrm{Au}, B \equiv \mathrm{Cu}$ and $A \equiv \mathrm{Au}$, $B^{\prime} \equiv$ Ag. The combination law was rather responsive to the physical parameters and carried out by resorting to well established solid-state data $[51,52,45,38], \widetilde{\mu}_{A}=1, \widetilde{\mu}_{B}=$ $0.99999, \widetilde{\mu}_{B^{\prime}}=0.99998 ; \sigma_{A}=58.8 \mathrm{~S} / \mu \mathrm{m}, \sigma_{B}=45.5$ $\mathrm{S} / \mu \mathrm{m}, \sigma_{B^{\prime}}=62.1 \mathrm{~S} / \mu \mathrm{m} ; n_{A}=59.0 \mathrm{~nm}^{-3}, n_{B}=84.7$ $\mathrm{nm}^{-3}, n_{B^{\prime}}=58.6 \mathrm{~nm}^{-3} ; \rho_{A}=19.28 \mathrm{~g} / \mathrm{cm}^{3}, \rho_{B}=8.96$ $\mathrm{g} / \mathrm{cm}^{3}, \rho_{B^{\prime}}=10.49 \mathrm{~g} / \mathrm{cm}^{3} ; \varepsilon_{A F}=5.53 \mathrm{eV}, \varepsilon_{B F}=7 \mathrm{eV}$, $\varepsilon_{B^{\prime} F}=5.49 \mathrm{eV}$. Then we obtain $\lambda_{A} \sqrt{\varepsilon_{A F}} \approx 0.49 d_{A}$, $\lambda_{B}^{B^{\prime} F} \sqrt{\varepsilon_{B F}} \approx 0.63 d_{B}, \lambda_{B^{\prime}} \sqrt{\varepsilon_{B^{\prime} F}} \approx 0.57 d_{B^{\prime}}$, with $d_{i}$ denoting here the effective thickness in $\mathrm{nm}$. On conforming to the real sample geometries, $d_{i}$ were set to 5 times the values in Table (1). As all films grew at constant surface area, mass probabilities read $p_{i}=\rho_{i} d_{i} /\left(\rho_{A} d_{A}+\rho_{B} d_{B}\right)$ (and identically in $B^{\prime}$ ). Effective Fermi's energy fractions $\alpha_{i}$ may depend on intensive and extensive film variables coming from the statistical thermodynamics of bulk and interface phases. To the authors' knowledge useful analytical expressions are unknown, but the orders of magnitude of all $\alpha_{i}$ must be small enough to preserve the validity of Eq. (13). Fermi's energies then should decrease with increasing layer thickness [53], an influence getting more pro- 


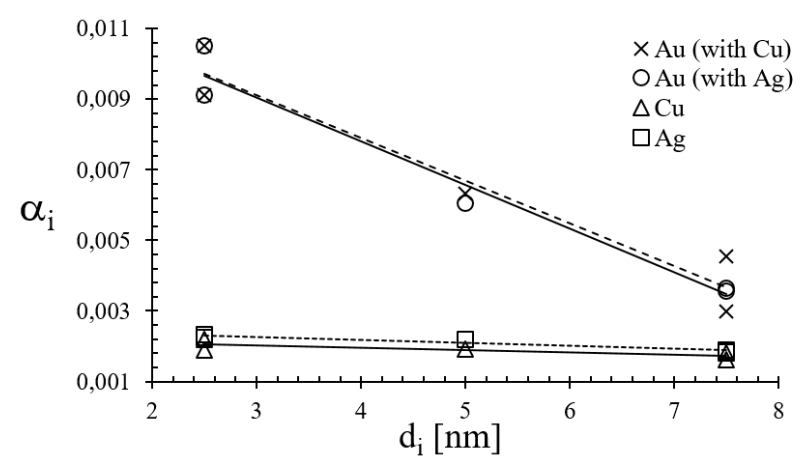

Figure 4 Fermi's energy fractions in $\mathrm{Cu} / \mathrm{Au}$ and $\mathrm{Au} / \mathrm{Ag}$ damping.

nounced as the size quantization level is approached. Accordingly, $\alpha$-terms were coupled in each metal pair to obey a linearly decreasing function of $d_{i}$, bearing in mind they may stand for some lowest-order expansion of a more complex dependence. To a good approximation, one has $\alpha_{A} \approx$ $\left(12.7-1.2 d_{A}\right) \cdot 10^{-3}, \alpha_{B} \approx\left(22-0.7 d_{B}\right) \cdot 10^{-4}(\mathrm{Cu} / \mathrm{Au})$, $\alpha_{A} \approx\left(12.8-1.2 d_{A}\right) \cdot 10^{-3}, \alpha_{B^{\prime}} \approx\left(25-0.8 d_{B}\right) \cdot 10^{-4}$ (Au/Ag), each term multiplying $d_{i}$ being $\mathrm{nm}^{-1}$. The found $\alpha_{i}$ vs. $d_{i}\left(i=A, B ; i=A, B^{\prime}\right)$, once inserted in Eq. (13), recover all damping energies in Table (1) with relative errors $<2.5 \cdot 10^{-4}$. Figure 4 suggests that $\alpha_{i}$ may stand for an optical solid-state property, since $\alpha_{A}$ does not change significantly in the two systems. Consistently with former assumptions, and the orders of magnitude of Drude's parameters, then we find $\alpha_{i} \varepsilon_{i F}<\gamma_{i}$ and $\alpha_{i} \varepsilon_{i F} \quad<<\omega_{i P}$, with mean values $\alpha_{A} \varepsilon_{A F} \approx 37 \mathrm{meV}(\mathrm{Au}), \alpha_{B} \varepsilon_{B F} \approx 13$ $\mathrm{meV}(\mathrm{Cu}), \alpha_{B^{\prime}} \varepsilon_{B^{\prime} F} \approx 15 \mathrm{meV}$ (Ag). Finally, to point out another proof of consistency, a layer coupling interaction $u=u(|\mathbf{r}|)$ was added to $\mathrm{U}$ in Eq. (2), distorting the displacements into:

$$
r_{i}^{*}=-\frac{q_{i} \mathrm{E}_{i} \pm \nabla_{\mathbf{r}} u_{\perp}}{m_{i} \omega_{i}\left(\omega_{i}+\mathrm{i} \gamma_{i}\right)},
$$

$\nabla_{\mathbf{r}} u_{\perp} \equiv \mathbf{e}_{\mathrm{t}} \cdot \nabla_{\mathbf{r}} u$ and the positive sign at the numerator referring (conventionally) to $A$. For a coupling term $\left\langle t_{i}\right\rangle \equiv$ $\pm \nabla_{\mathbf{r}} u_{\perp} / q_{i} \mathrm{E}_{i}$, Eq. (13) should be reconsidered upon:

$g_{A}^{*}=\left(1+\left\langle t_{A}\right\rangle\right) g_{A}, g_{B}^{*}=\left(1-\left\langle t_{B}\right\rangle\right) g_{B}, \bar{g}^{*}=\bar{g}^{*}\left(\left\langle t_{A}\right\rangle,\left\langle t_{B}\right\rangle\right)$

In fact the former analysis for $\mathrm{Cu} / \mathrm{Au}$ is formally equivalent to set $\alpha_{A}^{*}=1.27 \cdot 10^{-2}, \alpha_{B}^{*}=2.2 \cdot 10^{-3}$ and $\sqrt{1 \pm\left\langle t_{i}\right\rangle}=$ $\alpha_{i} / \alpha_{i}^{*}$, yielding $\left\langle t_{A}\right\rangle \approx\left(1-9.4 \cdot 10^{-2} d_{A}\right)^{2}-1$ and $\left\langle t_{B}\right\rangle \approx$ $1-\left(1-3.2 \cdot 10^{-2} d_{B}\right)^{2}$. Alike trends could be observed as well for $\mathrm{Au} / \mathrm{Ag}$. This again emphasizes the role of chemical specificity and the way nanolayers may couple via bulk or surface interactions.

Conclusions $\mathrm{e}^{-}$damping in multilayer thin $(\mathrm{Cu} / \mathrm{Au}$ and $\mathrm{Au} / \mathrm{Ag}$ ) films was measured and interpreted by linking a mean-field picture to the free $\mathrm{e}^{-}$model. The combination rule of single coefficients is of the parallel type and coupled to given Fermi's energy fractions in each metal.
Acknowledgements This work was supported by the Ministry of Environment and Energy, the Ministry of Science and Education, the Environmental Protection and Energy Efficiency Fund and the Croatian Science Foundation under the project Plasmonic Alternative Materials for Solar Energy Conversion" (PKP2016-06-4469) in the total amount of 1074000 HRK.

\section{References}

[1] Y. Hashimoto, G. Seniutinas, A. Balčytis, S. Juodkazis, and Y. Nishijima, Sci. Rep. 6, 25010 (2016).

[2] C. Gong and M.S. Leite, ACS Photonics 3(4), 507-513 (2016).

[3] M. Grzelczak, S. A. Mezzasalma, W. Ni, Y. Herasimenka, L. Feruglio, T. Montini, J. Prez-Juste, P. Fornasiero, M. Prato, and L. M. Liz-Marzn, Langmuir 28(24), 88268833 (2012).

[4] H. Kobayashi, K. Kusada, and H. Kitagawa, Accounts Chem. Res. 48(6), 1551-1559 (2015).

[5] M. G. Blaber, M. D. Arnold, and M. J. Ford, J. Phys.: Condens. Mat. 22(14), 143201 (2010).

[6] D. Rioux, S. Vallières, S. Besner, P. Muñoz, E. Mazur, and M. Meunier, Adv. Opt. Mater. 2(2), 176-182 (2014).

[7] J. Zhu, Physica E 27(1-2), 296-301 (2005).

[8] A. K. Samal, L. Polavarapu, S. Rodal-Cedeira, L. M. LizMarzan, J. Perez-Juste, and I. Pastoriza-Santos, Langmuir 29(48), 15076-15082 (2013).

[9] L. Yang, W. Yan, H. Wang, H. Zhuang, and J. Zhang, RSC Adv. 7(19), 11355-11361 (2017).

[10] D. Kosters, A. de Hoogh, H. Zeijlemaker, H. Acar, N. Rotenberg, and L. Kuipers, ACS Photonics 4(7), 18581863 (2017).

[11] A. J. Logsdail and R. L. Johnston, J. Phys. Chem. C 116(44), 23616-23628 (2012).

[12] Y. Song, K. Liu, and S. Chen, Langmuir 28(49), 17143 17152 (2012).

[13] H. A. Macleod, Thin-Film Optical Filters (CRC Press, Boca Raton, 2017).

[14] Z. Knittl, Optics of Thin Films: An Optical Multilayer Theory (John Wiley-Sons Inc., London, 1976).

[15] M. Wei, L. Xu, J. Shi, G. Pan, Z. Cao, and X. Meng, Appl. Phys. Lett. 106(1), 011604 (2015).

[16] L. S. Abdallah, S. Zollner, C. Lavoie, A. Ozcan, and M. Raymond, Thin Solid Films 571, 484-489 (2014).

[17] Y. Nishijima and S. Akiyama, Opt. Mater. Express 2(9), 1226-1235 (2012).

[18] R. L. Chern, C. C. Chang, and C. C. Chang, Phys. Rev. E 73(Mar), 036605 (2006).

[19] A. A. Reijnders, J. Hamilton, V. Britto, J. B. Brubach, P. Roy, Q. D. Gibson, R. Cava, and K. Burch, Phys. Rev. B 90(23), 235144 (2014).

[20] A. Galuza and A. Beznosov, Low Temp. Phys. 27(3), 216227 (2001).

[21] D. E. Aspnes, Thin Solid Films 89(3), 249-262 (1982).

[22] M. Moskovits, I. Srnová-Šloufová, and B. Vlčková, J. Chem. Phys. 116(23), 10435-10446 (2002).

[23] J. Sancho-Parramon, V. Janicki, M. Lončarić, H. Zorc, P. Dubček, and S. Bernstorff, Appl. Phys. A 103(3), 745748 (2011). 
[24] W. Theiß, The use of effective medium theories in optical spectroscopy, (Springer Berlin Heidelberg, Berlin, Heidelberg, 1993), pp. 149-176.

[25] G. V. Naik, V. M. Shalaev, and A. Boltasseva, Adv. Mater. 25(24), 3264-3294 (2013).

[26] G. V. Hartland, L. V. Besteiro, P. Johns, and A. O. Govorov, ACS Energy Lett. 2(-), 1641-1653 (2017).

[27] M. Gaudry, J. Lermé, E. Cottancin, M. Pellarin, J.L. Vialle, M. Broyer, B. Prével, M. Treilleux, and P. Mélinon, Phys. Rev. B 64(Aug), 085407 (2001).

[28] S. A. Mezzasalma and G. J. Koper, Colloid Polym. Sci. 280(2), 160-166 (2002).

[29] V. M. Anisimov, G. Lamoureux, I. V. Vorobyov, N. Huang, B. Roux, and A. D. MacKerell, J. Chem. Theory Comput. 1(1), 153-168 (2005).

[30] S. A. Mezzasalma, J. Chem. Phys. 113(8), 3434-3440 (2000).

[31] S. A. Mezzasalma, J. Phys. Chem. Solids 61(4), 593-601 (2000).

[32] P. B. Johnson and R. W. Christy, Physical Review B 6(12), 4370 (1972).

[33] M. Hövel, B. Gompf, and M. Dressel, Thin Solid Films 519(9), 2955-2958 (2011).

[34] J. Sancho-Parramon, V. Janicki, and H. Zorc, J. Nanophotonics 5(1), 051805 (2011).

[35] P. Melpignano, C. Cioarec, R. Clergereaux, N. Gherardi, C. Villeneuve, and L. Datas, Organic Electronics 11(6), 1111-1119 (2010).

[36] V. Holý, U. Pietsch, and T. Baumbach, High Resolution XRay Scattering from Thin Films and Multilayers (Springer Tracts in Modern Physics, vol. 149, 1999).

[37] E. J. Zeman and G. C. Schatz, J. Phys. Chem. 91(3), 634643 (1987).

[38] M. Fox, Optical Properties of Solids (Oxford University Press, Oxford, 2001).

[39] H. U. Yang, J. D' Archangel, M. L. Sundheimer, E. Tucker, G. D. Boreman, and M.B. Raschke, Physical Review B 91(23), 235137 (2015).

[40] K. P. Chen, V. P. Drachev, J. D. Borneman, A. V. Kildishev, and V. M. Shalaev, Nano letters 10(3), 916-922 (2010).

[41] J. D. Jackson, Classical Electrodynamics (John Wiley \& Sons Inc., New York, 1962).

[42] H. Goldstein, C. P. Poole, and J. L. Safko, Classical Mechanics (Pearson Education Limited, 2014).

[43] U. Kreibig and L. Genzel, Surface Science 156, 678 - 700 (1985).

[44] D. L. Goodstein, States of Matter (Dover Publications Inc., Toronto, 1985).

[45] N. W. Ashcroft and N.D. Mermin, Solid State Physics (Harcourt College Publishing, Fort Worth, 1976).

[46] O. Peña-Rodríguez, M. Caro, A. Rivera, J. Olivares, J. M. Perlado, and C. Alfredo, Opt. Mater. Express 4(2), 403410 (2014).

[47] H. L. Engquist and G. Grimvall, Phys. Rev. B 21(Mar), 2072-2077 (1980).

[48] L. Genzel, T. P. Martin, and U. Kreibig, Zeit. Phys. B Cond. Mat. 21(4), 339-346 (1975).

[49] E. A. Coronado and G. C. Schatz, J. Chem. Phys. 119(7), 3926-3934 (2003)

[50] E. Bringuier, Eur. J. Phys. 23(3), 367 (2002).
[51] D. R. e. Lide, CRC Handbook of Chemistry and Physics (CRC Press, Boca Raton, 2009).

[52] C. Kittel, Introduction to Solid State Physics (John Wiley \& Sons, Oxford, 1986).

[53] V. D. Dymnikov, Phys. Solid State 53(5), 901-907 (2011).

Copyright line will be provided by the publisher 